\title{
Investigation of miRNA expression profiles using cohort samples reveals potential early detectability of colorectal cancers by serum miR-26a-5p before clinical diagnosis
}

\author{
ASAHI HISHIDA ${ }^{1}$, HIROYA YAMADA ${ }^{2}$, YOSHITAKA ANDO ${ }^{3}$, YOSHINAGA OKUGAWA ${ }^{4,5}$, \\ MANABU SHIOZAWA $^{6}$, YOHEI MIYAGI ${ }^{7}$, YATARO DAIGO ${ }^{8,9}$, YUJI TOIYAMA $^{5}$, YUMIKO SHIRAI $^{10}$, \\ KOJI TANAKA $^{11}$, YOKO KUBO ${ }^{1}$, RIEKO OKADA ${ }^{1}$, MAKO NAGAYOSHI $^{1}$, TAKASHI TAMURA ${ }^{1}$, \\ ATSUYOSHI MORI $^{12}$, TAKAAKI KONDO ${ }^{13}$, NOBUYUKI HAMAJIMA ${ }^{14}$, KENJI TAKEUCHI ${ }^{1}$ and KENJI WAKAI ${ }^{1}$ \\ ${ }^{1}$ Department of Preventive Medicine, Nagoya University Graduate School of Medicine, Nagoya, Aichi 466-8550; \\ Departments of ${ }^{2}$ Hygiene and ${ }^{3}$ Informative Clinical Medicine, Fujita Health University School of Medical Sciences, \\ Toyoake, Aichi 470-1192; Departments of ${ }^{4}$ Genomic Medicine and ${ }^{5}$ Gastrointestinal and Pediatric Surgery, \\ Mie University Graduate School of Medicine, Tsu, Mie 514-8507; ${ }^{6}$ Department of Gastrointestinal Surgery, \\ Kanagawa Cancer Center Hospital; ${ }^{7}$ Molecular Pathology and Genetics Division, Kanagawa Cancer Center Research Institute, \\ Yokohama, Kanagawa $241-8515 ;{ }^{8}$ Center for Antibody and Vaccine Therapy, Institute of Medical Science, \\ Research Hospital, The University of Tokyo, Tokyo 108-8639; ${ }^{9}$ Department of Medical Oncology and Cancer Center, \\ Center for Advanced Medicine Against Cancer, Shiga University of Medical Science, Otsu, Shiga 520-2192; \\ Departments of ${ }^{10}$ Nutrition and ${ }^{11}$ Surgery, Iga City General Hospital, Iga, Mie 518-0823; \\ ${ }^{12}$ Seirei Preventive Health Care Center, Hamamatsu, Shizuoka 433-8558; \\ Departments of ${ }^{13}$ Pathophysiological Laboratory Sciences and ${ }^{14}$ Healthcare Administration, \\ Nagoya University Graduate School of Medicine, Nagoya, Aichi 466-8550, Japan
}

Received October 22, 2021; Accepted January 6, 2022

DOI: 10.3892/ol.2022.13207

\begin{abstract}
Previous studies have investigated the usefulness of microRNA (miRNA/miR) expression data for the early detection of colorectal cancer (CRC). However, limited data are available regarding miRNAs that detect CRC before clinical diagnoses. Accordingly, the present study investigated the early detectability of CRC by miRNAs using the preserved serum samples of the cohort participants affected with CRC within 2 years of study enrollment. First, the significant miRNAs were revealed using clinical CRC samples for a (seven early CRCs and seven controls) microarray analysis based on significance analysis of microarrays. Next, replicability was verified by reverse transcription-quantitative (RT-q) PCR (eight early CRCs and eight controls, together with 12 CRCs and 12 controls). Finally, early detectability was tested using the cohort samples of Japan Multi-Institutional
\end{abstract}

Correspondence to: Dr Asahi Hishida, Department of Preventive Medicine, Nagoya University Graduate School of Medicine, 65 Tsurumai-cho, Showa, Nagoya, Aichi 466-8550, Japan

E-mail: a-hishi@med.nagoya-u.ac.jp

Key words: microRNA, colorectal cancer, early detection of cancer, cohort study
Collaborative Cohort Study (17 CRCs and 17 controls) to reveal how a certain number of patients developed CRC within 2 years after participation. In the discovery phase, miRNA expression measurements were conducted using a 3D-Gene Human miRNA Oligo Chip for 2,555 miRNAs, and RT-qPCR analyses were performed to validate the replicability. In the first validation set with eight CRCs with early clinical stage and eight age- and gender-matched controls, miR-26a-5p and miR-223-3p demonstrated the highest diagnostic accuracy of area under the curve (AUC) $=1.000$ (sensitivity and specificity 100\%). In an examination of the predictability of CRC incidence using pre-clinical cohort samples, miR-26a-5p demonstrated good predictability of advanced CRC incidence with an AUC of 0.840. Overall, the present study revealed serum miR-26a-5p as a potential early detection marker for CRC.

\section{Introduction}

Colorectal cancer (CRC) is one of the commonest cancers worldwide, next to the cancers of lung and breast, while it continues to be among the top 3 cancers both in incidence and mortality in Japan $(1,2)$. The incidence of CRC is in an increasing trend with the Westernized lifestyle getting more and more prevalent in Japan, making it one of the most important public health burdens to countermeasure along with other issues such as infectious diseases (3). Accumulated clinical 
evidence shows that the life expectancy of CRC patients is favorable when their CRCs are found in early stage with 5 -year overall survival (OAS) rate of more than $84 \%$, whereas the OAS of advanced CRC patients are much lower, $60.0 \%$ for those with clinical stage IIIb and $18.8 \%$ for those with clinical stage IV, suggesting the importance of early detection of CRCs (4).

MicroRNAs (miRNAs) are small non-coding RNAs 20-25 nucleotides in length that play roles in transcriptional and post-transcriptional gene regulation in normal cells. MiRNAs have also been shown to play essential roles in the development and progression of various types of cancers (5). Recently, medical researchers worldwide made substantial efforts to clarify the roles of miRNAs in and their potential applicability to early detection of cancers, which have led to the accumulation of useful evidence for future development of clinical tests based on blood miRNA measurements in practical medicine (6-8).

Although there are a considerable number of studies that investigated the possible usefulness of miRNA expression data for early detection of CRCs (9-11), there is still little evidence that clarified the detectability of CRC onset by the miRNA expression analysis using the samples from cohort study participants who were before clinical diagnoses.

Accordingly, we set out to examine the early detectability of CRCs by serum miRNAs using the preserved serum samples of the cohort participants who were affected with CRC within 2 years after study enrollments.

\section{Materials and methods}

Study subjects. For the discovery phase, the discovery cohort included seven CRC patients who underwent surgery at Kanagawa Cancer Center Hospital between years of 2019 and 2020. In the first validation phase, the case subjects were 8 CRC patients who underwent surgery at Kanagawa Cancer Center Hospital between years of 2019 and 2020. In the second validation phase, the case subjects were $12 \mathrm{CRC}$ patients who visited or were admitted to Iga City General Hospital between years of 2014 and 2016. In the discovery and validation phases, the control subjects were age- and gender-matched cancer-free subjects from Iga City Cohort Study who participated between years of 2013 and 2014 without any history of cancer and without any cancer incidence at least within a year after participation, with one-to-one matching.

The test cohort included patients from the Japan Multi-Institutional Collaborative Cohort Study (J-MICC Study), one of the largest genome cohort studies in Japan with 92,610 participants who were recruited nationwide from 14 areas of Japan between 2005 and 2014 (12). We obtained informed consent from the participants in the J-MICC Study. We collected blood samples using a $7 \mathrm{ml}$ vacuum tube for serum and $7 \mathrm{ml}$ EDTA-2Na containing vacuum tube for plasma and buffy coat. We also conducted a survey using self-administered questionnaires to the participants that included items on drinking, smoking, physical activities, sleep, food consumption, medical history, and reproductive history.

For the test phase for early detectability of CRCs before clinical diagnosis, cases were the 17 participants of J-MICC
Shizuoka and Daiko Studies and Iga City Cohort Study, all of which are the sub-cohorts of the J-MICC Study, who developed CRCs within 2 years after participation, and the control subjects were age- and gender-matched cancer-free subjects from the corresponding cohorts (Shizuoka, Daiko and Iga City Cohort Studies) without any history of cancer and without any cancer incidence at least within a year after participation, with one-to-one matching. We first discovered the significantly up/down-regulated miRNAs using the clinical CRC samples (7 early CRCs and 7 controls in the 1st stage, discovery phase) by microarray analysis based on SAM (significance analysis of microarrays) (13). We next verified their replicability by real-time PCR ( 8 early CRCs and 8 controls, together with 12 early and advanced CRCs and 12 controls in the 2 nd stage, validation phase). Finally, we tested the early detectability using the cohort samples of J-MICC Shizuoka \& Daiko Studies $(14,15)$ who developed CRC within 2 years after participation (3rd stage, test phase) (Fig. 1). Written informed consent was obtained from all participants. The protocol of this study was approved by the Ethics Review Committee of the Nagoya University Graduate School of Medicine (approval no. 2019-0314-8), Aichi Cancer Center, Kanagawa Cancer Center and all the participated institutions.

Follow-up. In the J-MICC Study, we followed up the cancer incidence by the survey of regional cancer registries, where cancers were coded based on ICD-O-3T as C00-C80 (and CRCs were coded as C18-C20). For the follow-up of death, we conducted the survey on death certificates and classified the underlying causes of deaths based on ICD-10, where cancers were coded as C00-C97 (and CRCs were coded as C18-C20). Participants who moved out of the catchment areas were considered as dropouts, who were excluded from the follow-up survey after the timepoints of move-outs and treated as truncated.

MiRNA expression analysis with microarray. MiRNAs were extracted from $400 \mu \mathrm{l}$ of serum samples using the miRNeasy extraction kit (Qiagen). Prior to microarray analyses, the integrity of total RNA samples was checked by detecting the peak from small RNA fragments using the Agilent 2100 Bioanalyzer (Agilent Technologies). In the discovery phase, miRNA expression measurements were conducted using a 3D-Gene miRNA Labeling kit and a 3D-Gene Human miRNA Oligo Chip (Toray Industries Inc.) for the detection of the expressions of microRNA sequences from 271 organisms: 38,589 hairpin precursors and 48,860 mature microRNAs registered in the miRBase release 22 (www.mirbase.org). The raw data of each spot was normalized by substitution with a mean intensity of the background signal determined by all blank spots' signal intensities of $95 \%$ confidence intervals. Measurements of spots with the signal intensities greater than 2 standard deviations (SD) of the background signal intensity were considered to be valid. A relative expression level of a given miRNA was calculated by comparing the signal intensities of the valid spots throughout the microarray experiments. The normalized data were normalized per array, such that the 75th percentile of the signal intensity was adjusted to 1 . 


\section{Discovery phase}

(CRC stage I\&II [7 vs. 7; cases from Kanagawa cancer center, controls from Iga], microarray: SAM)

Validation phase

(early CRC [8 vs. 8; cases from Kanagawa cancer center, controls from Iga] \& early+advanced CRC [12 vs. 12; cases and controls from lga], real-time PCR: $\triangle \Delta C q$ )

Test phase

(cohort samples, $<2$ years before clinical diagnosis [17 incident CRCs vs. 17 controls from J-MICC], real-time PCR: $\Delta \Delta \mathrm{Cq}$ )

Figure 1. Workflow of the present study. CRC, colorectal cancer; SAM, significance analysis of microarrays; Ct, threshold cycles; J-MICC, Japan Multi-Institutional Collaborative Cohort.

Quantitative RT-PCR analysis. To validate the replicability of the miRNAs detected by microarray analyses, we conducted the quantitative RT-PCR (qRT-PCR) analyses. miRNAs were extracted from $200 \mu \mathrm{l}$ of serum samples using the miRNeasy Mini Kit (Qiagen). The miRNAs were then reverse transcribed using the miRCURY LNA RT Kit (Qiagen). The obtained cDNA was quantified by qRT-PCR in the ABI-PRISM 7900 Sequence Detection System and the StepOnePlus System (Applied Biosystems, Foster City, CA) using the miRCURY LNA miRNA PCR Kit (Qiagen) according to manufacturer's instructions (details of the primers used are shown in Table S1). The experiment was performed in duplicate and the average cycle threshold $(\mathrm{Cq})$ value was obtained. Quantification of miRNA expression levels was conducted based on the comparative cycle threshold $(\Delta \Delta \mathrm{Cq})$ method, where the expression levels of each miRNA were normalized based on the expression levels of miR-16-5p as an internal control (16). Two-tailed P-values are calculated based on a Student's t-test of the $2^{-\Delta \Delta \mathrm{Cq}}$ values for each gene comparing cases and controls. In the test phase for early detectability of CRCs before clinical diagnosis, experiments were conducted with blinding to examiners (whose names are in the Acknowledgements).

Database search. We referred to the databases of miRCancer (www.mircancer.ecu.edu) (17) to search for the previously reported miRNAs whose expressions were associated with the development or progression of CRCs.

Statistical analysis. The comparisons between the miRNA expression data of CRC patients and control subjects based on microarray measurements were conducted using SAM, using the 'samr' package of $\mathrm{R}$ ver. 3.6.3, where q-value is used as a means to control the positive false discovery rate in multiple hypothesis testing (18), after excluding the miRNAs with percent presence of the signals in either of cases or controls was less than $50 \%$. ROC curve analyses were conducted using the MedCalc ver. 20.011 (MedCalc Software Ltd.). Graphs for
miRNA expression levels were drawn using the 'beeswarm' package of $\mathrm{R}$. Comparisons of the two miRNA expression levels were conducted using the Student's t-test (unpaired, 2-sided) as well as by Mann-Whitney's U test, and those of more than three miRNA expression levels were conducted by one-way analysis of variance (ANOVA) with post hoc analysis by Tukey's test by using Stata (StataCorp).

\section{Results}

Study characteristics. The characteristics of the study subjects are shown in Table I. Briefly, the discovery set consisted of 7 early CRC cases and 7 age- and gender-matched controls, the first validation set consisted of 8 early CRC cases with 8 age- and gender-matched controls, and the second validation set consisted of $12 \mathrm{CRC}$ cases with mixture of early and advanced stages together with 12 age- and gender-matched controls, whereas the test set of cohort samples for pre-clinical early detection consisted of 17 cohort participants who developed CRC with mixture of early and advanced clinical stages within 2 years after participation, together with 17 age- and gender-matched controls.

Microarray miRNA expression analysis of CRC patients and controls. We conducted the miRNA expression analyses according to the workflow presented in Fig. 1. We selected the candidate miRNA based on the combined strategy of theoretical selection with best statistical significance and the empirical selection by database search (17) and our previous reports $(9,10)$. The comprehensive expression measurements of miRNAs by microarrays and subsequent SAM analysis revealed miR-6765, miR-5100, miR-572 (up-regulated) and miR-518e-5p (down-regulated) as the miRNAs with best discrimination abilities. In addition, we searched for the miRNAs with smallest q-values which were previously reported in association with CRCs among the miRNAs that fulfilled the criteria of FDR $<0.25$ (562 up-regulated miRNAs 
Table I. Characteristics of the study subjects.

\begin{tabular}{|c|c|c|c|c|c|c|c|c|}
\hline \multirow[b]{2}{*}{ Variables } & \multicolumn{2}{|c|}{ Discovery } & \multicolumn{2}{|c|}{ Validation-1 } & \multicolumn{2}{|c|}{ Validation-2 } & \multicolumn{2}{|c|}{ Test (cohort, J-MICC) } \\
\hline & $\begin{array}{l}\text { Case } \\
(n=7)\end{array}$ & $\begin{array}{c}\text { Control } \\
(n=7)\end{array}$ & $\begin{array}{l}\text { Case } \\
(n=8)\end{array}$ & $\begin{array}{c}\text { Control } \\
(n=8)\end{array}$ & $\begin{array}{l}\text { Case } \\
(n=12)\end{array}$ & $\begin{array}{l}\text { Control } \\
(n=12)\end{array}$ & $\begin{array}{c}\text { Case } \\
(n=17)\end{array}$ & $\begin{array}{l}\text { Control } \\
(n=17)\end{array}$ \\
\hline Male (\%) & $5(71.4 \%)$ & $5(71.4 \%)$ & $4(33.3 \%)$ & $4(33.3 \%)$ & $8(66.7 \%)$ & $8(66.7 \%)$ & $12(70.6 \%)$ & $12(70.6 \%)$ \\
\hline Age, mean $\pm \mathrm{SD}$ & $58.4 \pm 6.9$ & $58.0 \pm 5.7$ & $74.5 \pm 4.2$ & $64.5 \pm 1.3$ & $69.0 \pm 7.7$ & $63.8 \pm 4.5$ & $59.7 \pm 8.0$ & $59.5 \pm 7.3$ \\
\hline \multicolumn{9}{|l|}{ Cancer site } \\
\hline Colon $(\mathrm{C})$ & $0(0.0 \%)$ & - & $4(50.0 \%)$ & - & $2(16.7 \%)$ & - & $4(23.5 \%)$ & - \\
\hline Sigmoid (S) & $2(28.6 \%)$ & - & $1(12.5 \%)$ & - & $6(50.0 \%)$ & - & $7(41.2 \%)$ & - \\
\hline Rectum (R) & $5(71.4 \%)$ & - & $3(37.5 \%)$ & - & $4(33.3 \%)$ & - & $4(23.5 \%)$ & - \\
\hline $\mathrm{S}-\mathrm{R}$ & $0(0.0 \%)$ & - & $0(0.0 \%)$ & - & $0(0.0 \%)$ & - & $2(11.8 \%)$ & - \\
\hline \multicolumn{9}{|l|}{ Clinical stage } \\
\hline I & $6(85.7 \%)$ & - & $3(37.5 \%)$ & - & $1(8.3 \%)$ & - & $3(17.6 \%)$ & - \\
\hline II & $1(14.3 \%)$ & - & $5(62.5 \%)$ & - & $4(33.3 \%)$ & - & $7(41.2 \%)$ & - \\
\hline III & $0(0.0 \%)$ & - & $0(0.0 \%)$ & - & $7(58.3 \%)$ & - & $3(17.6 \%)$ & - \\
\hline IV & $0(0.0 \%)$ & - & $0(0.0 \%)$ & - & $0(0.0 \%)$ & - & $2(11.8 \%)$ & - \\
\hline (Unknown) & $0(0.0 \%)$ & - & $0(0.0 \%)$ & - & $0(0.0 \%)$ & - & $2(11.8 \%)$ & - \\
\hline
\end{tabular}

SD, standard deviation; S-R, sigmoid colon and rectum; J-MICC, Japan Multi-Institutional Collaborative Cohort; Validation-1, 1st validation set from Kanagawa Cancer Center; Validation-2, 2nd validation set from Iga City General Hospital.

Table II. Selected miRNAs in the discovery phase.

A, miRNAs upregulated in CRCs

\begin{tabular}{ll}
\hline miRNAs & Fold-change $\quad$ q-value $(\%)$
\end{tabular}

\begin{tabular}{lll}
\hline Smallest q-value & & \\
miR-6765-3p & 3.356 & 0 \\
$\operatorname{miR}-5100$ & 3.114 & 0 \\
$\operatorname{miR}-572$ & 3.152 & 0
\end{tabular}

CRC associated, smallest

q-value

$\begin{array}{lcc}\operatorname{miR}-223-3 p & 2.867 & 0 \\ \operatorname{miR}-26 a-5 p & 2.862 & 4.091\end{array}$

CRC associated, reported

by our group $(9,10)$

miR-21-5p

1.766

9.431

$\mathrm{B}$, miRNAs downregulated in CRCs

\begin{tabular}{lcc}
\hline miRNAs & Fold-change & q-value (\%) \\
\hline $\begin{array}{l}\text { Smallest q-value } \\
\text { miR-518e-5p }\end{array}$ & 0.381 & 15.197 \\
CRC associated, smallest & & \\
q-value & & \\
miR-22-5p & 0.578 & 15.197 \\
miR-15a-5p & 0.474 & 15.197 \\
\hline
\end{tabular}

CRC, colorectal cancer; miR, microRNA. and 255 down-regulated miRNAs), which led us to adopt miR-223-3p, miR-26a-5p and miR-21-5p as the up-regulated, miR-22-5p and miR-15a-5p as the down-regulated candidate miRNAs (Table II and Fig. 2). We also confirmed that there was no statistically significant difference in expression levels of miR-16-5p (the internal control) observed between cases and controls (Fig. S1).

Validation with quantitative RT-PCR analysis with independent samples. We next verified the replicability of microarray discovered miRNAs by qRT-PCR using independent samples. In the first validation set with $8 \mathrm{CRC}$ samples with early clinical stage from Kanagawa Cancer Center and 8 age- and gender-matched controls, miR-26a-5p and miR-223-3p demonstrated the highest diagnostic accuracy of $\mathrm{AUC}=1.000$ (sensitivity $100.0 \%$ and specificity $100.0 \%$ ), followed by miR-21-5p with AUC of 0.859 (sensitivity $100.0 \%$ and specificity 62.5\%) (Fig. 3A). In the second validation set with the independent set with 12 advanced CRCs and 12 controls from Iga City General Hospital, most of the selected miRs demonstrated moderate accuracy with AUCs of 0.6-0.8 (Fig. 3B). The expressions of the top 3 significant up-regulated miRNAs (miR-6765, miR-5100 and miR-572) and the top significant down-regulated miRNA (miR-518e-5p) were not detected in the qRT-PCR of the validation phase. The expression levels of replicated miRNAs (miRNAs with AUC of more than 0.6 and the same directions of correlations as those in the discovery phase) as well as those of the rest of the miRNAs that failed to replicate are shown in Fig. 4.

Examination of the predictability of CRC incidence using pre-clinical cohort samples. We next examined the predictabilities of CRC incidence by the replicated miRNAs that 


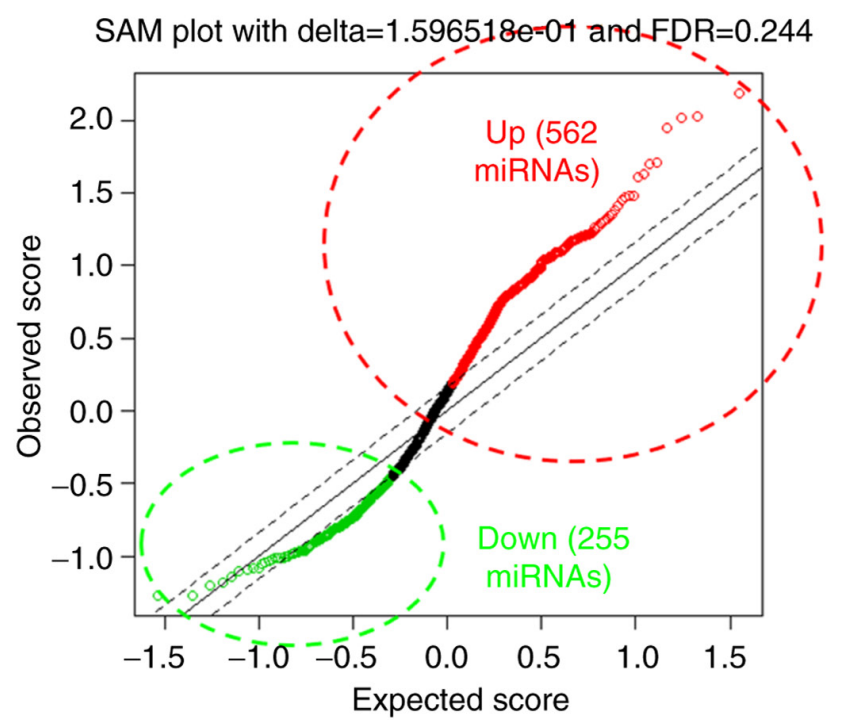

(Top significant miRNAs)

miR-6765-3p

$\operatorname{miR}-5100$

miR-572

miR-518e-5p

(CRC-associated miRNAs)

miR-223-3p

miR-21-5p

miR-22-5p

(SE-miRNAs in CRC) (Suzuki HI, Cell 2017)

miR-26a-5p

$\operatorname{miR}-15 a-5 p$

Figure 2. SAM plot for significant miRNA discovery and selected miRNAs for validation. Upregulated miRNAs are shown in red and downregulated miRNAs are shown in green. Top significant miRNAs are miRNAs with smallest q-values (18) in SAM. miRNAs/miRs, microRNA; SAM, significance analysis of microarrays; CRC, colorectal cancer; SE-miRNAs in CRC, super-enhancer-associated miRNAs in colorectal cancer (23).

demonstrated the AUC of more than 0.6 in both of the two validation phases with the same direction of correlations between expression levels of miRNAs and CRC risk as those in the discovery phase 17 samples from patients from the J-MICC Shizuoka and Daiko Studies $(14,15)$ who developed CRCs within 2 years after participation and age- and gender-matched controls.

Among the two miRNAs that fulfilled the criteria (miR-26a-5p and miR-223-3p), miR-26a-5p demonstrated the good predictability of advanced CRC incidence (5 incident CRCs and 5 age- and gender-matched controls) with the AUC of 0.840 (sensitivity $100.0 \%$ and specificity $60.0 \%$ ), whereas no other miRs fulfilled the criteria of AUC more than 0.6, and in the same direction of correlations as those in the discovery and validation sets (Figs. 5 and S2). The predictability of early CRC by miR-26a-5p and miR-223-3p did not reach the AUC of 0.6 (Fig. S3). The expression levels of miR-26a-5p and miR-223-3p in the pre-clinical phase are shown in Supplementary Fig. S2. In addition, we compared the expression levels of miR-26a-5p between early CRC, advanced CRC, and non-CRC subjects, which revealed statistically significant difference $(\mathrm{P}=0.049$, one-way ANOVA) (Fig. S4).

\section{Discussion}

In the present study, on the basis of discovery and validation with clinical CRC samples and testing with pre-clinical cohort samples, we identified serum miR-26a-5p as a possible early detection marker for CRC before clinical diagnosis.

With regard to the roles of miR-26a-5p in the colorectal carcinogenesis, considerable amount of experimental evidence has been accumulated. The expressions of miR-26a have been reportedly inversely correlated with PTEN expressions in human CRC tissue samples, where miR-26a was significantly upregulated in tumors in comparison to normal tissues, and there was no difference in miR-26a expression levels by CRC stages (19). MiR-26a is also demonstrated to downregulate retinoblastoma 1 gene expression and also to regulate glucose metabolism by targeting $P D H X$ in CRCs (20).

Meanwhile, miR-223-3p is shown to promote the proliferation, growth, migration, invasion and EMT (epithelial-mesenchymal transition) of CRCs through the negative regulation of PRDMI (21). Although the present study failed to detect the early detectability of CRCs by miR-223-3p, the high accuracy of CRC detectability in the validation phase may indicate its importance in the development of CRCs. MiR-21 is also shown to be a robust diagnostic marker for CRCs, which failed to detect CRC incidence before clinical diagnosis. Considering that the high expression of miR-21 in serum and CRC tissue is associated with larger tumor size, distant metastasis and poor prognosis, as demonstrated in the previous papers $(10,22)$, the role of miR-21 in the early detection of CRCs might be relatively limited.

In addition, miR-26a-5p is one of the super-enhancer-associated miRNAs (SE-miRNAs) of CRCs, where super-enhancers (SEs) are newly found regulatory regions on the genome consisted of multiple enhancer-like elements occupied with high densities of master transcription factors and mediator complexes (23). Moreover, both miR-15a and miR-26a-5p are marked with super-enhancers in normal sigmoid colon and other normal tissues (23), thus the roles of these SE-miRNAs in the normal colorectal tissues as well as in the development of CRCs should be clarified experimentally and clinically in the future. Further clarification of the roles of SE-miRNAs in CRC development as well as in the early detection of CRCs should be warranted.

In the present study, the detectability of selected miRNAs in the discovery phase was improved in the first validation set with CRC cases of early clinical stages compared to the second validation set with CRC cases of mixture of early and advanced clinical stages, which was in accordance with the findings with the previous report of miRNA diagnostic model in lung cancer, although the difference in diagnostic accuracies between early and advanced stage of 
A
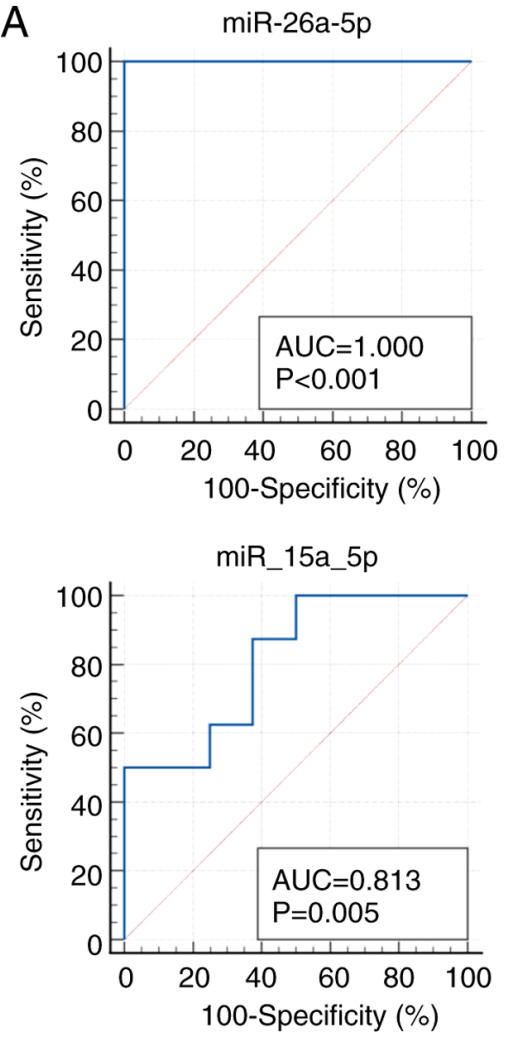

B

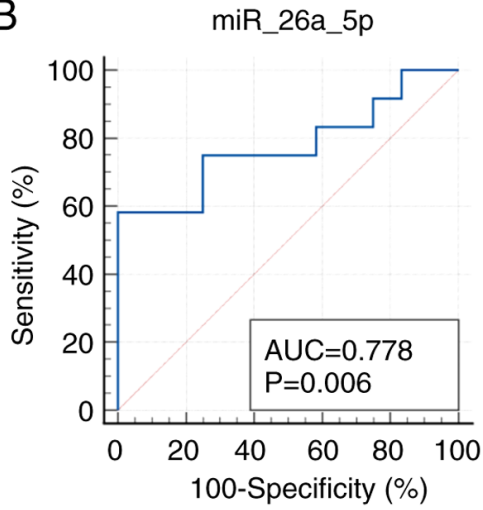

miR-15a-5p

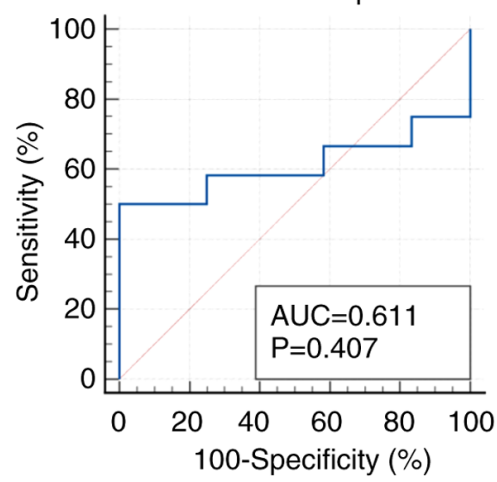

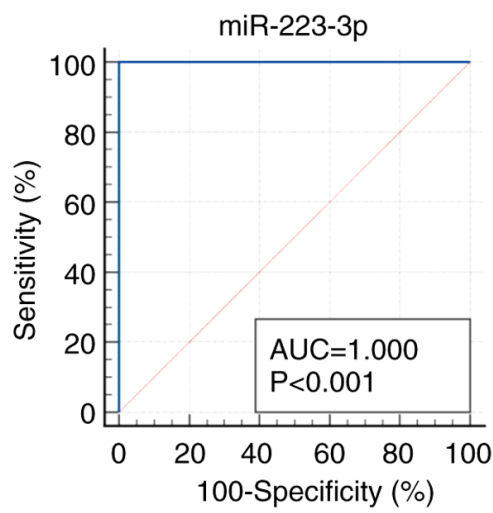
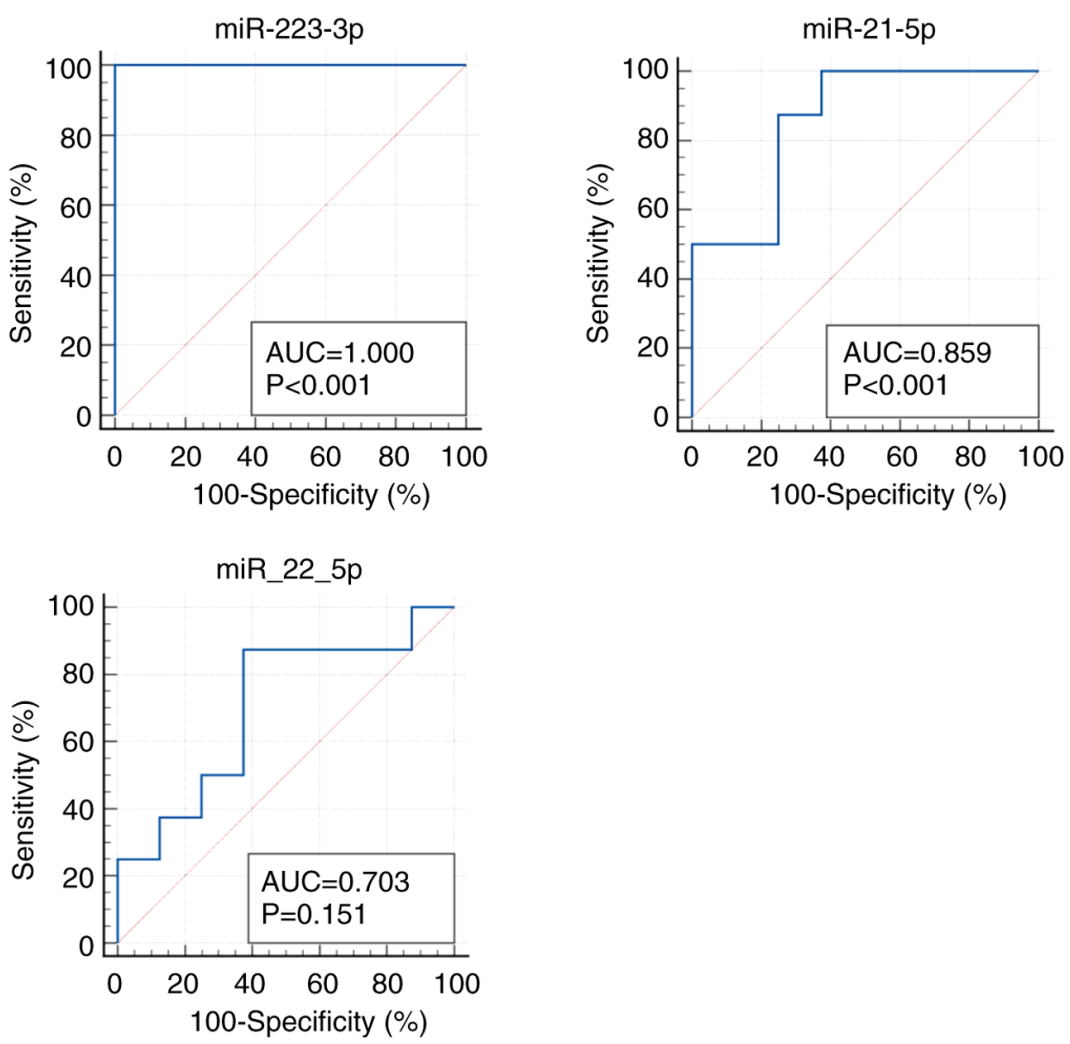

miR_223_3p
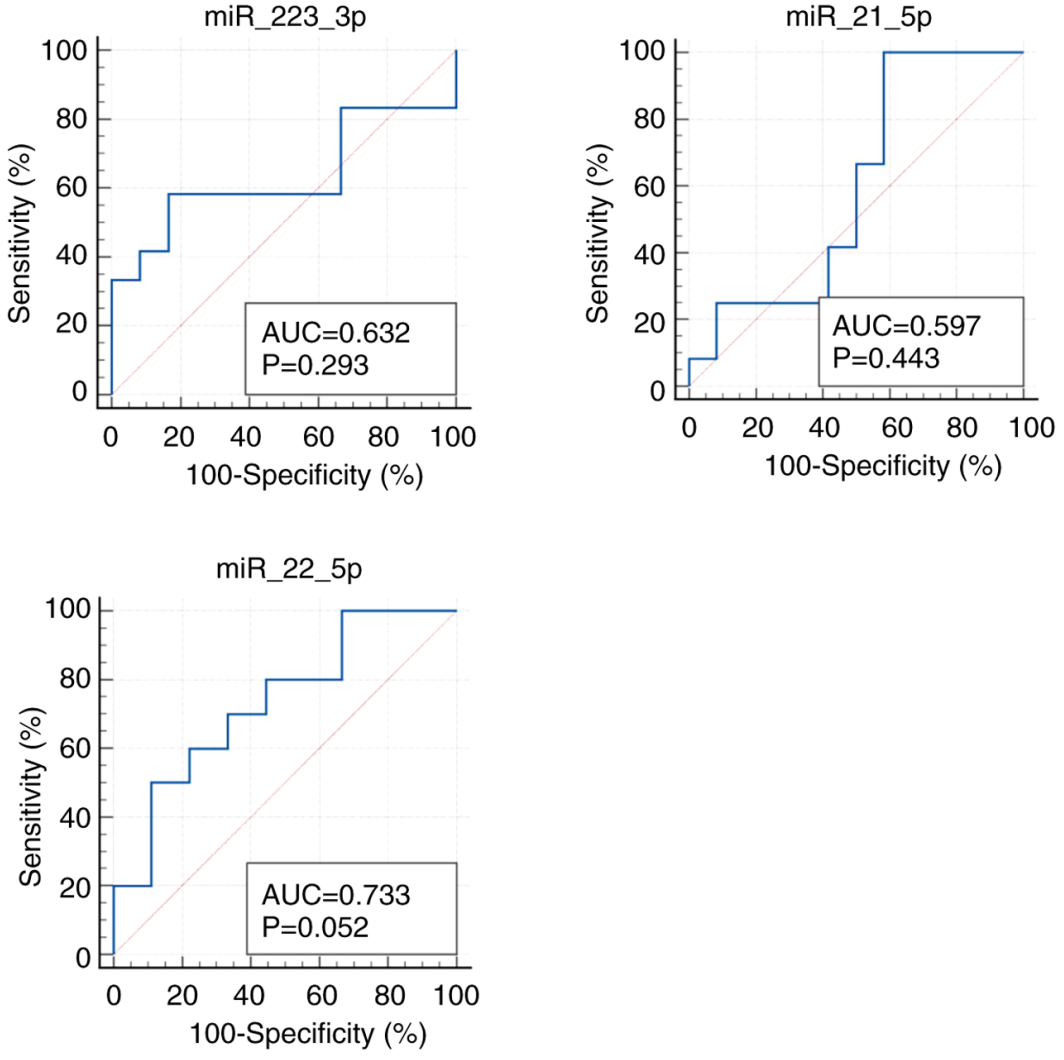

Figure 3. ROC curves in the validation data set. (A) 1st validation set from Kanagawa. (B) 2nd validation set from Iga. CRC, colorectal cancer; miRNA/miR, microRNA; AUC, area under the curve; ROC curve, receiver operating characteristic curve.

lung cancers was only a little (24). It also remains unclear whether the changes in miRNA expression levels reflect the presence of diseases, i.e., the existence of cancers itself, or reflect the alterations of surrounding tissues such as microenvironments, and it is neither clarified whether the miRNA expression changes initiates prior to disease onset 
A
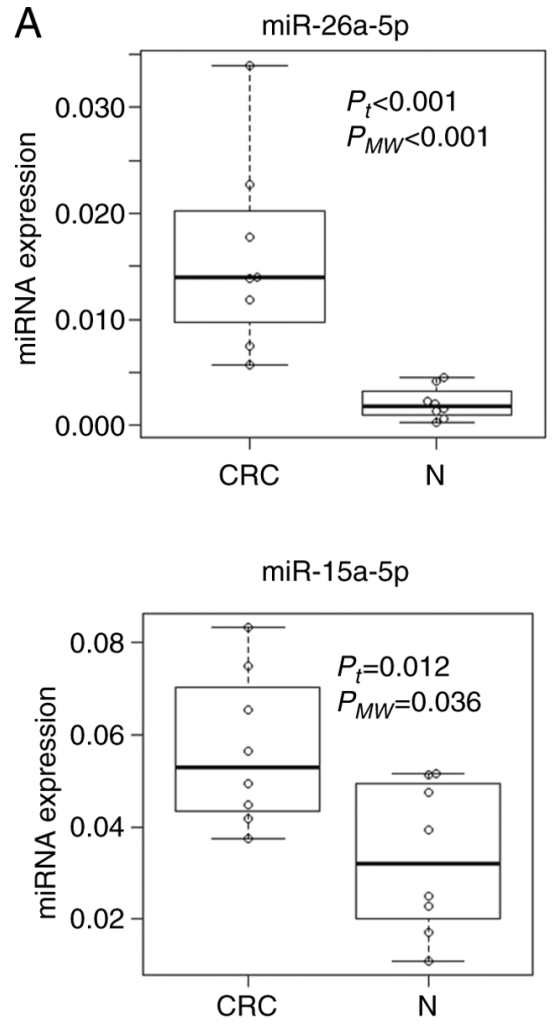

B
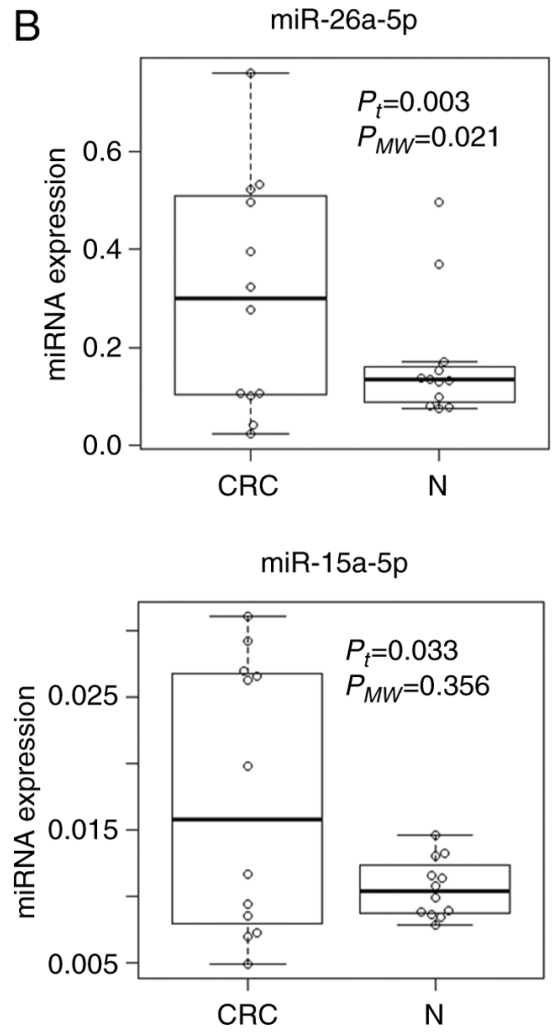
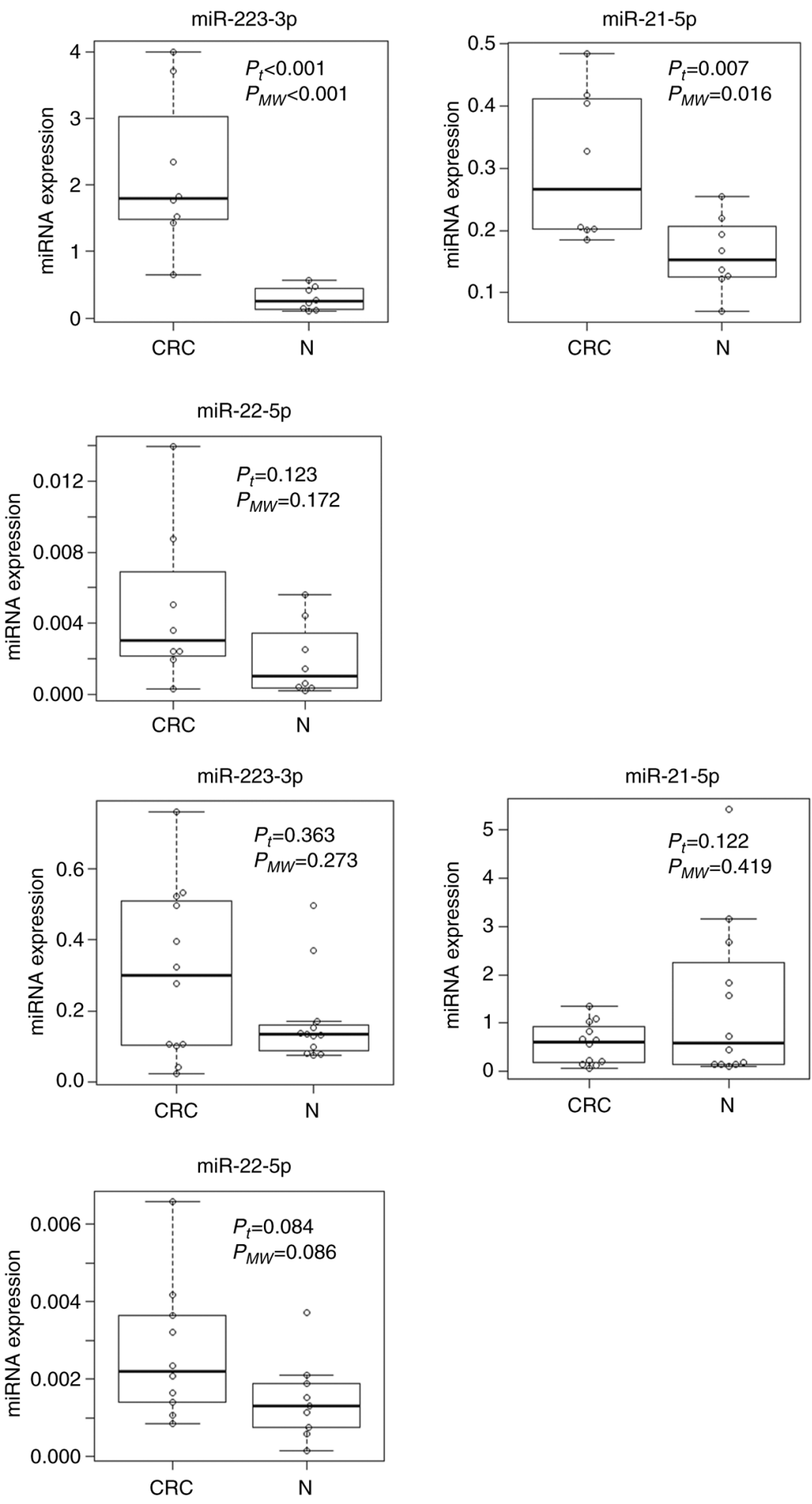

Figure 4. Expression levels of replicated miRNAs in the validation data set. (A) 1st validation set from Kanagawa. (B) 2nd validation set from Iga. Two-tailed P-values are calculated based on an unpaired Student's t-test of the $2^{-\Delta \Delta C q}$ values for each gene comparing cases and controls. CRC, colorectal cancer; miRNA/miR, microRNA; $P_{\mathrm{t}}$, P-value for t-test; $P_{\mathrm{MW}}, \mathrm{P}$-value for Mann-Whitney's U test; N, normal.

or not (25). Further biological and epidemiological studies are warranted.

One strength to be mentioned of the present study would be the availability of serum samples of cohort participants who developed CRCs after participation, which enabled us to evaluate the early detectability of CRCs before clinical diagnosis. Further studies that will make use of the preserved samples of large cohort studies for early detection of cancers might be expected. 


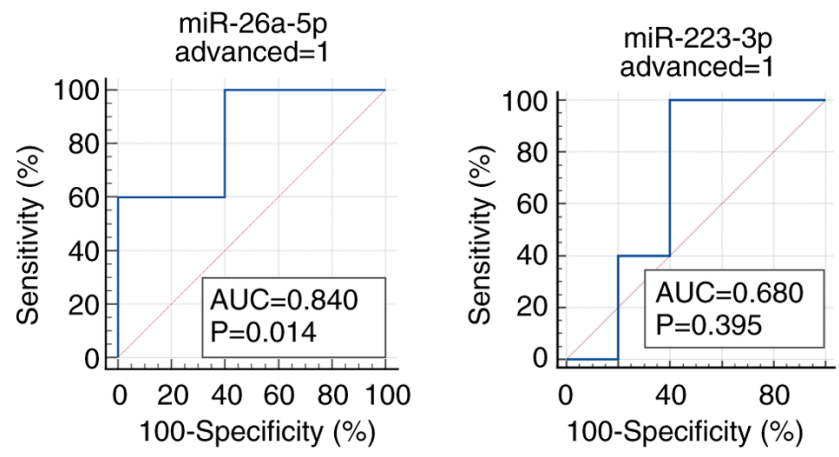

Figure 5. Early detectability of colorectal cancer before clinical diagnosis in cohort participants by ROC curves. ROC curves were generated using the pre-clinical cohort samples (five advanced CRCs and five controls). CRC, colorectal cancer; miRNA/miR, microRNA; AUC, area under the curve; ROC curve, receiver operating characteristic curve; advanced, advanced colorectal cancer.

There are several limitations to be mentioned in the present study. Although the present study analyzed the frozen samples preserved for several years for early detectability of CRC, the miRNAs are shown to be highly stable for $\sim 5$ years under the storage at $-80^{\circ} \mathrm{C}(26)$. To obtain more reliable results, however, further studies with improved designs, e.g., those with more frequent follow-ups with blood sample collections will be expected. In the present study, miRNA expression levels in the serum were measured. Although the miRNA expression levels in serum and plasma are in good correlations to each other, there are some advantages and disadvantages. For example, whereas the miRNA expression levels in serum tends to be higher due to the RNA release during coagulation (27), the miRNA expression levels in plasma might also be affected by delayed sample processing, especially in cohort studies, due to the possible release of exosomes from the blood cells. In addition, aberrant expressions of miR-26a-5p in blood have been reported also in acute myeloid leukemia (AML) (28), bladder cancer $(29,30)$, non-small cell lung cancer (31) and breast cancer (32). Although non-specificity is recognized as a general problem of cancer detection markers, it is worth reporting the present study results considering miR-26a-5p can be one of the potential markers for future clinical applications. Further investigations should be made to identify more specific early detection methods of CRCs. There were also constraints due to limited research funds; we had to select the limited number of miRNAs with high accuracy carefully, although adopting as numerous miRNAs as possible to improve the diagnostic accuracy of miRNA prediction models would be ideal. In addition, some of the miRNAs detected in the discovery phase indicated poor reproducibility of expressions themselves in the validation phase, which was considered to be attributable to the performance of the microarray platform used. Therefore, studies with improved ways of expression analyses, i.e., by using the next-generation sequencings of miRNAs should be expected. Finally, statistical power considerations are as follows. The statistical power for the 10 -fold increase in expression levels with the standard deviation of 5 in 8 cases and 8 controls is more than $90 \%$, whereas it is more than $85 \%$ in 7 cases and 7 controls under the same conditions. Although the sample size in the present study can detect the difference in expression levels if the difference is large enough, the limited sample sizes of the present study should be considered as a limitation, which should warrant further investigations with sufficient sample sizes in the near future.

In conclusion, the present study found serum miR-26a-5p as a potential early detection marker for CRC before clinical diagnosis. Further investigations with improved designs are warranted.

\section{Acknowledgements}

The authors would like to thank Dr Nobuyuki Hamajima (Department of Healthcare Administration, Nagoya University Graduate School of Medicine) and Dr Hideo Tanaka (Kishiwada Healthcare Center) for their work for initiating and organizing the J-MICC Study as former principal investigators. The authors would also like to thank Ms. Yoko Mitsuda and Ms. Rie Terasawa (both affiliated with the Department of Preventative Medicine, Nagoya University Graduate School of Medicine) for their technical assistance.

\section{Funding}

This study was supported by Grants-in-Aid for Scientific Research for Priority Areas of Cancer (grant no. 17015018) and Innovative Areas (grant no. 221S0001) and by the Japan Society for the Promotion of Science (JSPS) KAKENHI grant from the Japanese Ministry of Education, Culture, Sports, Science and Technology [grant nos. 16H06277 (CoBiA) and 19K10598].

\section{Availability of data and materials}

The datasets used and/or analyzed during the current study are available from the corresponding author on reasonable request. The microarray data is publicly available from GEO (www.ncbi.nlm.nih.gov/geo/; accession no. GSE186510).

\section{Authors' contributions}

AH designed the study, analyzed the data and prepared the manuscript. HY and YA performed the experiments and prepared the data. YO, MS, YM, YD, YT, YS, KoT, YK, RO, MN, TT, KeT, AM, NH, TK and KW collected the data and samples. YM, YD, NH and KW supervised the study. AH and HY confirmed the authenticity of all the raw data All authors have read and approved the final manuscript.

\section{Ethics approval and consent to participate}

All of the patients agreed to provide their genetic and clinical data for analysis after written informed consent. The study protocol was approved by the Ethics Review Committee of the Nagoya University Graduate School of Medicine (approval no. 2019-0314-8), Aichi Cancer Center, Kanagawa Cancer Center and all the participated institutions.

\section{Patient consent for publication}

All study participants approved the publication of the present study. 


\section{Competing interests}

The authors declare that they have no competing interests.

\section{References}

1. Onyoh EF, Hsu WF, Chang LC, Lee YC, Wu MS and Chiu HM The rise of colorectal cancer in Asia: Epidemiology, screening, and management. Curr Gastroenterol Rep 21: 36, 2019.

2. Akimoto N, Ugai T, Zhong R, Hamada T, Fujiyoshi K Giannakis M, Wu K, Cao Y, Ng K and Ogino S: Rising incidence of early-onset colorectal cancer-a call to action. Nat Rev Clin Oncol 18: 230-243, 2021

3. Moore MA, Sobue T, Kuriki K, Tajima K, Tokudome S and Kono S: Comparison of Japanese, American-Whites and African-Americans-pointers to risk factors to underlying distribution of tumours in the colorectum. Asian Pac J Cancer Prev 6 : 412-419, 2005

4. Hashiguchi Y, Muro K, Saito Y, Ito Y, Ajioka Y, Hamaguchi T, Hasegawa K, Hotta K, Ishida H, Ishiguro M, et al: Japanese society for cancer of the colon and rectum. Japanese society for cancer of the colon and rectum (JSCCR) guidelines 2019 for the treatment of colorectal cancer. Int J Clin Oncol 25: 1-42, 2020.

5. Goodall GJ and Wickramasinghe VO: RNA in cancer. Nat Rev Cancer 21: 22-36, 2021.

6. Abe S, Matsuzaki J, Sudo K, Oda I, Katai H, Kato K, Takizawa S, Sakamoto H, Takeshita F, Niida S, et al: A novel combination of serum microRNAs for the detection of early gastric cancer. Gastric Cancer 24: 835-843, 2021.

7. Yokoi A, Matsuzaki J, Yamamoto Y, Yoneoka Y, Takahashi K Shimizu H, Uehara T, Ishikawa M, Ikeda SI, Sonoda T, et al: Integrated extracellular microRNA profiling for ovarian cancer screening. Nat Commun 9: 4319, 2018.

8. Shimomura A, Shiino S, Kawauchi J, Takizawa S, Sakamoto H, Matsuzaki J, Ono M, Takeshita F, Niida S, Shimizu C, et al: Novel combination of serum microRNA for detecting breast cancer in the early stage. Cancer Sci 107: 326-334, 2016.

9. Yamada A, Horimatsu T, Okugawa Y, Nishida N, Honjo H, Ida $H$, Kou T, Kusaka T, Sasaki Y, Yagi M, et al: Serum miR-21-5p, miR-29a, and miR-125b are promising biomarkers for the early detection of colorectal neoplasia. Clin Cancer Res 21: 4234-4242, 2015.

10. Toiyama Y, Takahashi M, Hur K, Nagasaka T, Tanaka K, Inoue Y, Kusunoki M, Boland CR and Goel A: Serum miR-21-5p as a diagnostic and prognostic biomarker in colorectal cancer J Natl Cancer Inst 105: 849-859, 2013.

11. Toiyama Y, Okugawa Y, Fleshman J, Boland CR and Goel A: MicroRNAs as potential liquid biopsy biomarkers in colorectal cancer: A systematic review. Biochim Biophys Acta Rev Cancer 1870: 274-282, 2018.

12. Takeuchi K, Naito M, Kawai S, et al: Study profile of the Japan multi-institutional collaborative cohort (J-MICC) study. J Epidemiol 31: 660-668 2021.

13. Tusher VG, Tibshirani R and Chu G: Significance analysis of microarrays applied to the ionizing radiation response. Proc Natl Acad Sci USA 98: 5116-5121,2001.

14. Asai Y, Naito M, Suzuki M, Tomoda A, Kuwabara M, Fukada Y, Okamoto A, Oishi S, Ikeda K, Nakamura T, et al: Baseline data of Shizuoka area in the Japan multi-institutional collaborative cohort study (J-MICC Study). Nagoya J Med Sci 71: 137-144, 2009.

15. Morita E, Hamajima N, Hishida A, Aoyama K, Okada R, Kawai S, Tomita K, Kuriki S, Tamura T, Naito M, et al: Study profile on baseline survey of Daiko Study in the Japan multi-institutional collaborative cohort study (J-MICC Study). Nagoya J Med Sci 73: 187-195, 2011.

16. Schwarzenbach H, da Silva AM, Calin G and Pantel K: Data normalization strategies for MicroRNA quantification. Clin Chem 61: 1333-1342, 2015.
17. Xie B,Ding Q,Han Hand Wu D: miRCancer: A microRNA-cancer association database constructed by text mining on literature. Bioinformatics 29: 638-644, 2013.

18. Storey JD: The positive false discovery rate: A Bayesian interpretation and the q-value. Ann. Statist 31: 2013-2035, 2003

19. Coronel-Hernández J, López-Urrutia E, Contreras-Romero C, Delgado-Waldo I, Figueroa-González G, Campos-Parra AD, Salgado-García R, Martínez-Gutierrez A, Rodríguez-Morales M, Jacobo-Herrera N, et al: Cell migration and proliferation are regulated by miR-26a-5p in colorectal cancer via the PTEN-AKT axis. Cancer Cell Int 19: 80, 2019.

20. López-Urrutia E, Coronel-Hernández J, García-Castillo V, Contreras-Romero C, Martínez-Gutierrez A, Estrada-Galicia D, Terrazas LI, López-Camarillo C, Maldonado-Martínez H, Jacobo-Herrera N and Pérez-Plasencia C: MiR-26a downregulates retinoblastoma in colorectal cancer. Tumour Biol: Apr 26 , 2017 (Epub ahead of print). doi: 10.1177/1010428317695945

21. Chai B, Guo Y, Cui X, Liu J, Suo Y, Dou Z and Li N: MiR-223-3p promotes the proliferation, invasion and migration of colon cancer cells by negative regulating PRDM1. Am J Transl Res 11: 4516-4523, 2019

22. Okugawa Y, Yao L, Toiyama Y, Yamamoto A, Shigemori T, Yin C, Omura Y, Ide S, Kitajima T, Shimura T, et al: Prognostic impact of sarcopenia and its correlation with circulating miR-21 in colorectal cancer patients. Oncol Rep 39: 1555-1564, 2018

23. Suzuki HI, Young RA and Sharp PA: Super-enhancer-mediated RNA processing revealed by integrative MicroRNA network analysis. Cell 168: 1000-1014.e15, 2017.

24. Asakura K, Kadota T, Matsuzaki J, Yoshida Y, Yamamoto Y, Nakagawa K, Takizawa S, Aoki Y, Nakamura E, Miura J, et al: A miRNA-based diagnostic model predicts resectable lung cancer in humans with high accuracy. Commun Biol 3: 134, 2020.

25. Yamada H, Suzuki K, Fujii R, Kawado M, Hashimoto S, Watanabe Y, Iso H, Fujino Y, Wakai $\mathrm{K}$ and Tamakoshi A: Circulating miR-21-5p, miR-29a, and miR-126 are associated with premature death risk due to cancer and cardiovascular disease: The JACC study. Sci Rep 11: 5298, 2021.

26. Balzano F, Deiana M, Giudici SD, Oggiano A, Baralla A, Pasella S, Mannu A, Pescatori M, Porcu B, Fanciulli G, et al: miRNA stability in frozen plasma samples. Molecules 20: 19030-19040, 2015.

27. Wang K, Yuan Y, Cho JH, McClarty S, Baxter D and Galas DJ: Comparing the MicroRNA spectrum between serum and plasma. PLoS One 7: e41561, 2012.

28. Barrera-Ramirez J, Lavoie JR, Maganti HB, Stanford WL, Ito C, Sabloff M, Brand M, Rosu-Myles M, Le Y, Allan DS, et al: Micro-RNA profiling of exosomes from marrow-derived mesenchymal stromal cells in patients with acute myeloid leukemia: Implications in leukemogenesis. Stem Cell Rev Rep 13: 817-825, 2017.

29. Wang $\mathrm{H}, \mathrm{Hu} \mathrm{Z}$ and Chen L: Enhanced plasma miR-26a-5p promotes the progression of bladder cancer via targeting PTEN. Oncol Lett 16: 4223-4228, 2018.

30. Miyamoto K, Seki N, Matsushita R, Yonemori M, Yoshino H, Nakagawa M and EnokidaH: Tumour-suppressive miRNA-26a-5p and miR-26b-5p inhibit cell aggressiveness by regulating PLOD2 in bladder cancer. Br J Cancer 115: 354-363, 2016.

31. Ye MF, Lin D, Li WJ, Xu HP and Zhang J: MiR-26a-5p serves as an oncogenic MicroRNA in non-small cell lung cancer by targeting FAF1. Cancer Manag Res 12: 7131-7142, 2020.

32. Huang ZM, Ge HF, Yang CC, Cai Y, Chen Z, Tian WZ and Tao JL: MicroRNA-26a-5p inhibits breast cancer cell growth by suppressing RNF6 expression. Kaohsiung J Med Sci 35: 467-473, 2019. International (CC BY-NC-ND 4.0) License. 